\title{
The flavone apigenin blocks nuclear translocation of sterol regulatory element-binding protein-2 in the hepatic cells WRL-68
}

\author{
Tsz Yan Wong ${ }^{1}$, Shu-Mei $\operatorname{Lin}^{2}$ and Lai K. Leung ${ }^{1,3 *}$ \\ ${ }^{1}$ Food and Nutritional Sciences Programme, School of Life Sciences, Faculty of Science, The Chinese University of Hong Kong, \\ Room 507C, MMW Building, Shatin, NT, Hong Kong \\ ${ }^{2}$ Department of Food Science, National Chiayi University, Chiayi City, Taiwan, ROC \\ ${ }^{3}$ Biochemistry Programmes, School of Life Sciences, Faculty of Science, The Chinese University of Hong Kong, Shatin, \\ NT, Hong Kong
}

(Submitted 8 January 2015 - Final revision received 10 March 2015 - Accepted 26 March 2015 - First published online 15 May 2015)

\section{Abstract}

Sterol regulatory element-binding protein-2 (SREBP-2) is a pivotal transcriptional factor in cholesterol metabolism. Factors interfering with the proper functioning of SREBP-2 potentially alter plasma lipid concentrations. Consuming fruits and vegetables is associated with beneficial plasma lipid profile. The mechanism by which plant foods induce desirable lipid changes remains unclear. Apigenin, a common plant food flavonoid, was shown to modulate the nuclear translocation of SREBP-2 in the hepatic cells WRL- 68 in the present study. The processing of SREBP-2 protein occurred after translation, and apigenin blocked this activation route. Further examination indicated that AMP-activated protein kinase (AMPK) was activated by the flavone, and co-administrating the AMPK-specific inhibitor compound $\mathrm{C}$ could release the blockage. Reporter gene assay revealed that the transactivation of sterol responsive element (SRE)-containing 3-hydroxy-3-methylglutaryl-CoA reductase (HMGCR) promoter was suppressed by the flavone. Similarly, electromobility shift assay result also demonstrated a reduced DNA-binding activity on the SRE domain under the same treatment. The reduced transactivity and DNA-binding activity could be attributed to a decreased amount of SREBP-2 translocating from cytosol to nucleus as depicted by confocal microscopy. Quantitative RT-PCR assay demonstrated that the transcription of HMGCR followed the same pattern of SREBP-2 translocation. In summary, the present study showed that apigenin prevented SREBP-2 translocation and reduced the downstream gene HMGCR transcription. The minimum effective dosage should be achievable in the form of functional food consumption or dietary supplementation.

\section{Key words: Apigenin: Sterol regulatory element-binding protein-2: 3-Hydroxy-3-methylglutaryl-CoA reductase:} Nuclear translocation

CVD is one of the leading causes of morbidity and mortality in the world. It is well-established that serum cholesterol level is negatively correlated with the risk of CVD. A metaanalysis has estimated that a drop of $100 \mathrm{mg} / \mathrm{dl}$ plasma cholesterol can reduce the mortality of $\mathrm{CHD}$ by $9 \%$ in the elderly $^{(1)}$. Cholesterol homeostasis is tightly controlled in human beings by the sterol regulatory element-binding protein (SREBP). SREBP-2 regulates 3-hydroxy-3-methylglutaryl-CoA reductase (HMGCR) expression, which catalyses the rate-limiting step of cholesterol biosynthesis. HMGCR inhibitors are prescribed clinically in treating patients with hypercholestrolemia. Thus, influencing HMGCR activity through SREBP-2 could be an alternative approach to treating this disease.
SREBP are basic helix-loop-helix-leucine zipper (bHLH-Zip) family transcriptional factors that regulate lipid metabolism ${ }^{(2)}$. Three subtypes - 1a, 1c and 2 - have been identified in this membrane-bound transcriptional factor family. The type $1 \mathrm{c}$ isoform appears to be involved in fatty acid and glucose metabolism, whereas the type 2 isoform mainly regulates cholesterol biosynthesis. Although the 1a isoform controls all SREBP responsive genes, it is not predominantly expressed in the liver.

Under normal physiological condition, SREBP-2 regulates cholesterol homeostasis by acting on the related target genes $^{(3)}$. When SREBP-2 is overexpressed ectopically, it enhances the expression of twelve enzymes involved in cholesterol biosynthesis ${ }^{(4)}$, and HMGCR is its prime target ${ }^{(5)}$.

Abbreviations: AMPK, AMP-activated protein kinase; HMGCR, 3-hydroxy-3-methylglutaryl-CoA reductase; LDLR, LDL receptor; SRE, sterol responsive element; SREBP, sterol regulatory element-binding protein.

*Corresponding author: Dr L. K. Leung, fax +852 26037732, email laikleung@cuhk.edu.hk 
The rate of cholesterol biosynthesis is increased by approximately 28 -fold in transgenic mice overexpressing SREBP-2 ${ }^{(2)}$.

SREBP-2's precursor form $(125 \mathrm{kDa})$ is encoded by the SREBP2 gene, and is activated by sterol regulatory elementbinding protein cleavage-activating protein (SCAP) in a post-translational modification like the other SREBP family members. Under sterol-deficient status, SCAP interacts with SREBP-2, and binds to the coatamer protein II vesicle. The complex then migrates from the endoplasmic reticulum to the Golgi. Site- 1 protease and Site- 2 protease in the organelle cut the SREBP-2 precursor sequentially to release the active transcriptional factor. The cleaved SREBP-2 (approximately $68 \mathrm{kDa}$ ) is then translocated to the nucleus, and binds to the sterol responsive element (SRE) of target genes. Under high sterol conditions, cholesterol binds to the sterolsensing domain of SCAP. Then SCAP undergoes conformational changes and binds to insulin-induced proteins (INSIG-1, -2) instead of SREBP; as a result, the nuclear translocation of SREBP-2 is reduced ${ }^{(2,6,7)}$.

SREBP-2 can be regulated at the levels of transcription and post-translation. Some signal transduction pathways can be involved along the process. The activation of phosphatidylinositol 3-kinase and Akt facilitates the transport of SREBP-2 to the Golgi for processing. Insulin-activated extracelluar signal-regulated protein kinase $1 / 2$ (ERK 1/2) can directly phosphorylate SREBP-2, and potentiate its transactivity ${ }^{(8)}$. In contrast, AMP-activated protein kinase (AMPK) phosphorylates the precursor factor, and prevents it from processing into its active form ${ }^{(9)}$. In addition, nuclear-bound SREBP-2 is subjected to ubiquitination and degradation in the cytosolic $26 \mathrm{~S}$ proteasome. This process is independent of the cholesterol status, while glycogen synthase kinase 3-mediated SREBP phosphorylation promotes the degradation ${ }^{(10)}$.

Dietary flavonoids have drawn much attention to their favourable effect on blood cholesterol. They are a group of plant pigments with a phenylchoromane or flavone ring ${ }^{(11)}$. Their benefit on hypercholesterolemia and CVD has been implicated in many studies. A cross-sectional study ${ }^{(12)}$ on Japanese women has demonstrated that increased flavonoid intake is associated with reduced plasma total cholesterol and LDL concentrations. Two meta-analyses have also shown that isoflavone intake is inversely correlated with the plasma LDL-cholesterol and $\mathrm{TAG}^{(13,14)}$.

Apigenin, or $4^{\prime}, 5,7$-trihydroxyflavone, is a common dietary flavonoid. It can be isolated from plant foods like apples, endive, beans, broccoli, celery, cherries, cloves, grapes, leeks, onions, barley, parsley and tomatoes; besides, tea and wine are plant-derived beverages containing apigenin ${ }^{(15)}$. It is considered as a chemopreventive agent due to its inhibitory effect on mutation ${ }^{(16)}$, oxidation ${ }^{(17,18)}$, inflammation and cell proliferation $^{(19)}$. Apigenin-rich extracts from Chamomile ${ }^{(20)}$ and celery have a long history in folk medicine prescribed as a remedy for heart attack.

In the present study, we hypothesised that some flavonoids isolated from fruits and vegetables were capable of controlling cholesterol synthesis by way of SREBP-2 intervention.

\section{Materials and methods}

\section{Chemicals}

Apigenin was obtained from Indofine Chemical. All other chemicals, if not stated otherwise, were acquired from Sigma Chemicals.

\section{Cell culture}

WRL-68 cells (American Type Culture Collection) were cultured in Roswell Park Memorial Institute (RPMI)-1640 media (Sigma Chemicals) with 10\% foetal bovine serum (Invitrogen Life Technology) and incubated at $37^{\circ} \mathrm{C}$ and $5 \% \mathrm{CO}_{2}$. These cells were routinely subcultured when reaching $80 \%$ of confluency. At $1 \mathrm{~d}$ before the experiment, the cultures were switched to RPMI-1640 phenol red free media (Sigma Chemicals) and 5\% charcoal-dextran treated foetal bovine serum (Hyclone). Subconfluent cell cultures were treated with various concentrations of apigenin with dimethyl sulphoxide as the carrier solvent. The final concentration of the solvent was $0 \cdot 1 \% \mathrm{v} / \mathrm{v}$, and the control cultures received dimethyl sulphoxide only. The cell density in each experiment was maintained at $5 \times 10^{2}$ cells $/ \mathrm{mm}^{2}$.

\section{Quantitative real time RT-PCR assay}

WRL-68 cells were seeded in six-well Costar plates, and they underwent various treatments. After $24 \mathrm{~h}$, total RNA was extracted from the cells using TRIzol reagent (Invitrogen). The concentration and purity of the RNA were determined by its absorbance at 260/280 nm. First DNA strands were synthesised from $3 \mu \mathrm{g}$ of total RNA, using oligo-dT primers and Moloney murine leukaemia virus RT (USB Corporation). Target fragments were quantified by real-time PCR, and an ABI StepOne Real-time PCR System (Applied Biosystems) was employed for these assays. Taqman ${ }^{\circledR} / \mathrm{VIC}^{\circledR}$ MGB probes and primers for SREBP-2, HMGCR, 3-hydroxy-3-methylglutaryl-CoA synthase (HMGCS), squalene epoxidase (SQLE), LDL receptor $\left(L D L R\right.$ ) and GAPDH (Assay-on-Demand ${ }^{\mathrm{TM}}$ ) and Real-time PCR Taqman Universal PCR Master Mix were all obtained from Applied Biosystems. PCR were set up as described in the manual, which were validated by the company. Signals obtained for GAPDH served as a reference to normalise the amount of RNA amplified in each reaction. Relative gene expression was analysed using the $2^{-\Delta \Delta C_{\mathrm{T}}} \operatorname{method}^{(21)}$.

\section{Luciferase reporter gene assay}

A fragment from human HMGCR 5'-flanking region (-1194 to -49 ) was amplified from human genomic DNA by using primers (forward: CGGGGTACCACCCTCCCTTTCTACCTTGTG and reverse: CCGCTCGAGACTTTCCTGTGCGAACCTTAC). The PCR product was digested with KpnI and XhoI and subcloned into a firefly luciferase reporter vector pGL3 (Clontech).

WRL-68 cells were seeded in ninety-six-well plates. After $24 \mathrm{~h}$, the cells were transiently transfected with $0 \cdot 25 \mu \mathrm{g}$ of the HMGCR promoter-driven firefly luciferase reporter plasmid and $3.0 \mathrm{ng}$ of Renilla luciferase control vector (Promega) in LipofectAmine (Invitrogen Life Technologies). 
After $6 \mathrm{~h}$, the medium was removed, and the cells were treated with various concentrations of apigenin for $24 \mathrm{~h}$. The cells were lysed, and the luciferase substrates (provided in DualLuciferase Assay Kit; Promega) were mixed with the cell lysate. The luciferase bioluminescence was measured by a FLUOstar Galaxy plate reader as described in the manual of the assay kit. The HMGCR transactivation activities represented by firefly luciferase light units were then normalised by that of Renilla luciferase.

\section{Electromobility shift assay}

Nuclear protein extract was isolated by using NucBuster $^{\mathrm{TM}}$ protein extraction kit (Novagen ${ }^{\circledR}$; EMD Biosciences, Inc.). In brief, cells were washed, trypsinised and centrifuged at $500 \mathrm{~g}$ at $4{ }^{\circ} \mathrm{C}$. Reagent 1 was added to the packed cells. Nuclear extract was isolated from the cell suspension by vortexing and centrifugation. The nuclear protein was stored at $-80^{\circ} \mathrm{C}$ until assayed. An oligonucleotide mimicking (-160 to - 141) HMGCR (5'-gtt ggc cga gcc cgt ggt gag aga tgg tgc ggt gCc tgt tct tgg $-3^{\prime}$ (SRE italicised) and $7 \times$ SRE: $5^{\prime}$-gtg cgg tgg tgc ggt ggt gcg gtg gtg cgg tgg tgc ggt ggt gcg gtg gtg cgg $\operatorname{tg}-3^{\prime}$ ) was synthesised and labelled by DIG Gel Shift Kit, 2nd Generation (Roche Diagnostics GmbH).

The nuclear protein was incubated with the labelled probe, sonicated salmon sperm DNA, poly(dI-dC) and binding buffer ( $400 \mathrm{~mm}-\mathrm{KCl}, \quad 80 \mathrm{~mm}$-HEPES, $2 \mathrm{~mm}$-dithiothreitol, $0.8 \mathrm{~mm}$-EDTA, $80 \%$ glycerol, $\mathrm{pH} 8$ ) provided in electrophoretic mobility shift assay accessory kit (Novagen) for $30 \mathrm{~min}$ at room temperature. $7 \times$ SRE unlabelled oligonucleotide or SREBP-2 antibody was co-incubated as the competitive control. The reaction mix was then separated on a $4-6 \%$ non-denaturing gel in $0.5 \times$ Tris-borate EDTA at $100 \mathrm{~V}$. The labelled oligonucleotideprotein complex was electro-transferred to a Nylon membrane, fixed by UV light, blocked and washed. The shifted oligonucleotide was detected by anti-Digoxigenin-AP conjugate and the chemiluminescent substrate CSPD ${ }^{\circledR}$ provided in the kit.

\section{Western blot analysis}

Cells were washed by PBS ( $\mathrm{pH} 7 \cdot 4$ ), and harvested into a $1.5 \mathrm{ml}$ microtube with $0.5 \mathrm{ml}$ lysis buffer (PBS, $1 \% \mathrm{NP} 40,0.5 \%$ sodium deoxycholate, $0 \cdot 1 \% \mathrm{SDS}$ ). The lysis buffer contained protease inhibitors $(40 \mathrm{mg} / 1$ phenylmethylsuphonyl fluoride, $0.5 \mathrm{mg} / 1$ aprotinin, $0.5 \mathrm{mg} / \mathrm{l}$ leupeptin, $1.1 \mathrm{~mm}$-EDTA and $0.7 \mathrm{mg} / \mathrm{l}$ pepstatin) and phosphatase inhibitor cocktail (PhosphoSTOP tablet; Roche). The harvested cells were then lysed with a cell disruptor (Branson Ultrasonics Corporation) on ice for $30 \mathrm{~s}$. The protein concentration of cell lysate was determined by Dc protein assay (BioRad). A total of $50 \mu \mathrm{g}$ of lysate protein were separated on $10 \%$ SDS-PAGE, and transferred onto an Immobilon PVDF membrane (Millipore). Primary antibodies of SREBP2 , phospho-extracelluar signal-regulated protein kinase $1 / 2$ (p-ERK 1/2), phospho-c-Jun N-terminal kinase (p-JNK) (catalogue no. SC-13552, SC-7383, SC-6254, respectively; Santa Cruz Biotechnology), phospho-p38, AMPK $\alpha$, phospho$\mathrm{AMP} \alpha$ (catalogue no. 9221, 2535, 2532, respectively; Cell Signaling Technology, Inc.), N-terminal SREBP-2 (catalogue no. AB30682; Abcam plc), HMGCR (catalogue no. ABS229; Millipore), actin (catalogue no. A-5316; Sigma Chemicals) and secondary antibodies conjugated with horseradish peroxidase (anti-rabbit HRP (catalogue no. SC-2004; Santa Cruz Biotechnology); anti-mouse HRP (catalogue no. 401215; Millipore)) were used for protein detection. An ECL Detection Kit (Amersham) provided the chemiluminescence substrate for horseradish peroxidase, and the targeted protein was visualised by autochemiluminography.

For the nuclear and cytosolic protein preparations, the NucBuster ${ }^{\mathrm{TM}}$ protein extraction kit $\left(\right.$ Novagen ${ }^{\circledR}$ ) was used as described earlier.

\section{Immunocytochemical imaging}

WRL-68 cells were grown on $35 \mathrm{~mm}$ glass bottom dishes, and were treated with $10 \mu \mathrm{M}$-apigenin at $40-50 \%$ confluence for $24 \mathrm{~h}$. After the treatment period, the cells were fixed with $4 \%$ paraformaldehyde in PBS with $0 \cdot 2 \%(\mathrm{v} / \mathrm{v})$ Tween 20 for $5 \mathrm{~min}$, followed by blocking in $3 \%$ bovine serum albumin PBS for $30 \mathrm{~min}$ at room temperature. The dishes were washed and incubated with anti-SREBP-2 and anti-golgin-97 primary antibody (1:100 dilution in PBS) for $3 \mathrm{~h}$. Subsequently, 1-h incubation of Alexa Fluor 488-labelled (Molecular Probes) and Alexa Fluor 568-labelled (Molecular Probes) secondary antibodies was carried out. Dishes were stained with 2-(4-amidinophenyl)-1H-indole-6-carboxamidine (DAPI), and the cells were examined by confocal microscopy.

\section{Statistical methods}

A Prism ${ }^{\circledR} 5.0$ (GraphPad Software, Inc.) software package was utilised for statistical analysis. Results were analysed by

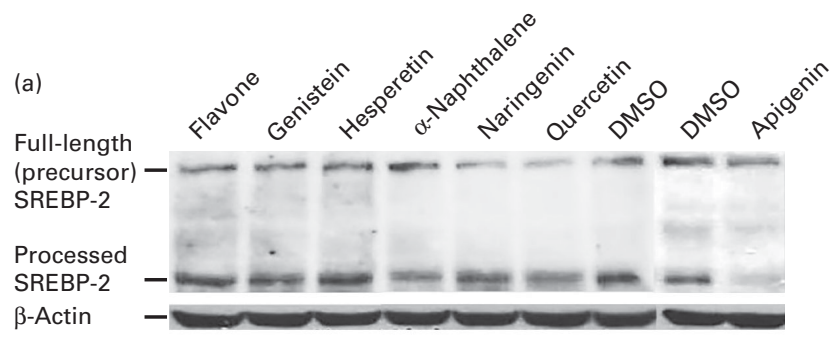

(b)

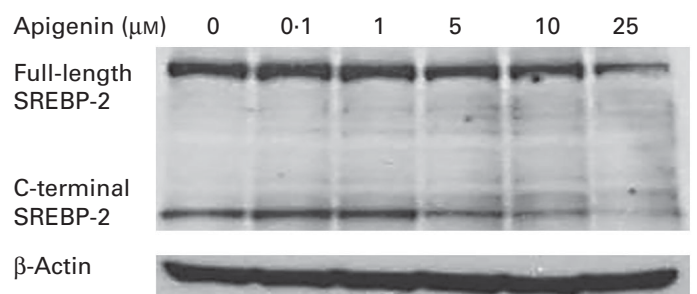

Fig. 1. The effect of flavonoids on sterol regulatory element-binding protein-2 (SREBP-2) processing. The hepatic cells WRL-68 were seeded in six-well culture plates and treated with various flavonoids at $25 \mu \mathrm{M}$. After $24 \mathrm{~h}$ of treatment, the cells were lysed and SREBP-2 processing was determined (a). A dose-response experiment was performed on apigenin as a follow-up to the screening (b). Apigenin was administered at $0,0 \cdot 1,1,5,10$ and $25 \mu \mathrm{M}$. DMSO, dimethyl sulphoxide. 
ANOVA and significant level was set at $P<0 \cdot 05$. The built-in Bonferroni post-test on selected pairs was performed when the significance was satisfied.

\section{Results}

\section{Effect of flavonoids on sterol regulatory element-binding} protein-2 processing in hepatic cells

SREBP-2 protein processing was determined in WRL-68 cells treated with various flavonoids as in Fig. 1(a). Given the same treatment concentration at $25 \mu \mathrm{m}$ for all compounds, apigenin appeared to be the most potent in impeding the processing of precursor SREBP-2. A dose-response experiment was then carried out in total cell lysates for cultures treated with apigenin, and a drop in the processed band intensity was observed at about $5 \mu \mathrm{M}$ as in Fig. 1(b). The precursor form or full-length of SREBP-2 would be cleaved into the $\mathrm{C}$ - and N-terminal fragments, and the N-fragment or N-SREBP-2 was the active transcriptional factor migrating into the nucleus and binding to SRE of genes. Analysis on the nuclear and cytosolic distribution of SREBP-2 revealed that reduced N-SREBP-2 was found in the nucleus under apigenin treatment (Fig. 2(a)). The optical density results (Fig. 2(b)) also indicated that the expression of total SREBP-2, full length plus C-SREBP-2, was also reduced at $25 \mu \mathrm{M}$-apigenin treatment.

\section{Immunocytochemical staining of sterol regulatory element-binding protein-2 protein}

As the processed SREBP-2 was reduced, we determined the translocation of the transcriptional factor. Contrasting to the

(a)

\section{Apigenin ( $\mu \mathrm{m})$ SREBP-2 \\ $\begin{array}{ll}\overline{0} & \text { C-terminal } \\ \text { O } & \text { SREBP-2 } \\ \text { ¿े } & \text { SREB }\end{array}$}

0

$0 \cdot 1$

1

5

10

25

GAPDH
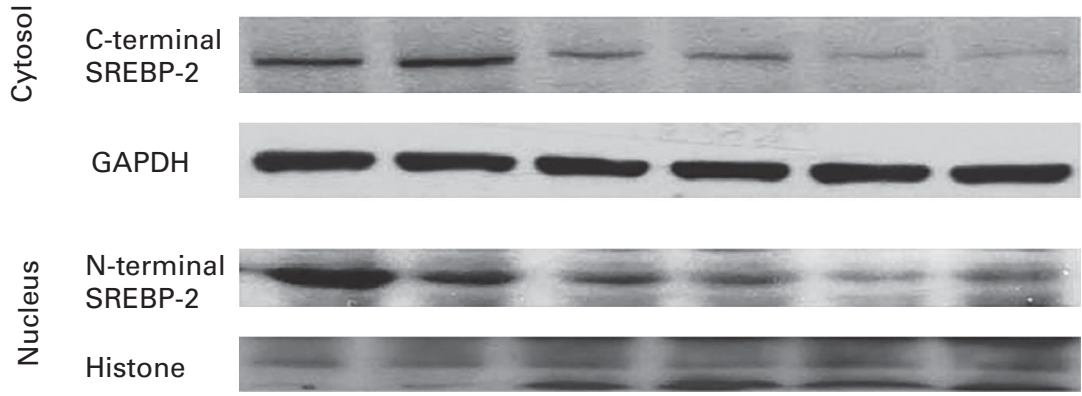

(b)
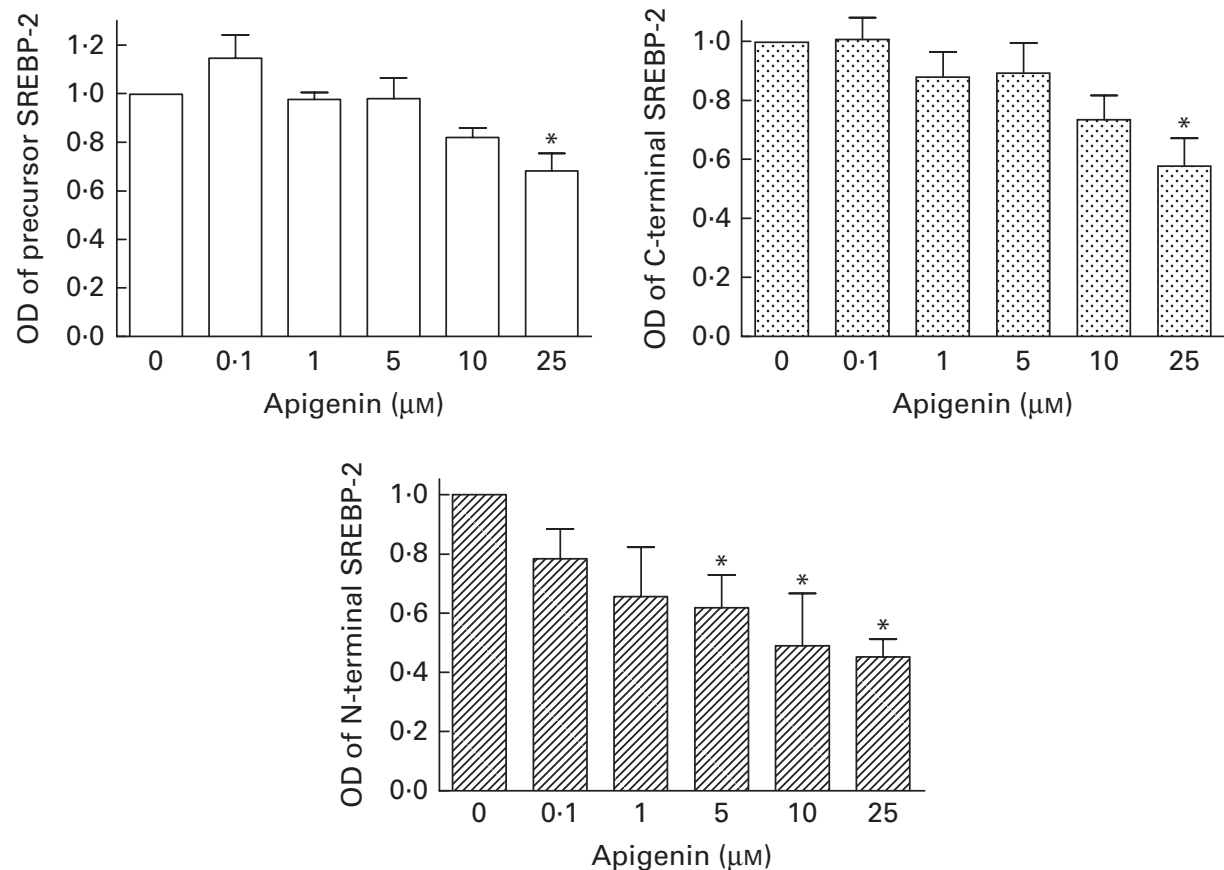

Fig. 2. Cytosolic and nuclear distribution of sterol regulatory element-binding protein-2 (SREBP-2). WRL-68 cells were cultured and were treated with apigenin. After $24 \mathrm{~h}$ of treatment, the extracts from nuclei and cytosolic fractions were immunoblotted for SREBP-2. In (a), images for cytosolic and nucleic SREBP-2 are displayed in the upper and lower panels, respectively. The optical density (OD) readings are shown in (b). Values are means, with their standard errors represented by vertical bars $(n 3)$. ${ }^{*}$ Mean value was significantly different $(P<0.05)$. 


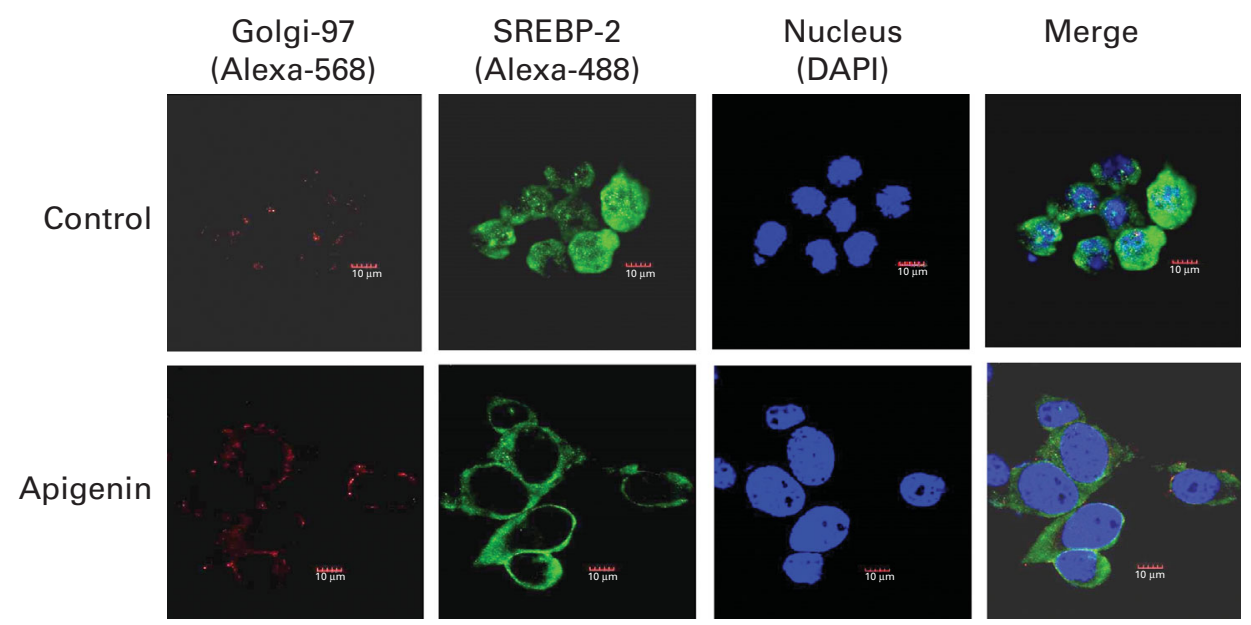

Fig. 3. Nuclear translocation of sterol regulatory element-binding protein-2 (SREBP-2) in apigenin-treated WRL-68 cells. The hepatic cells WRL-68 were seeded in six-well culture plates and treated with apigenin at $25 \mu \mathrm{M}$. After $24 \mathrm{~h}$ of treatment, the cells were fixed and incubated with Golgi-specific and SREBP-2 primary antibodies and fluorophore (Alexa 568 and 488)-labelled secondary antibodies. The nuclei were counterstained with 2-(4-amidinophenyl)-1H-indole-6-carboxamidine (DAPI). The images were taken by confocal microscopy. The image is a representation of two independent experiments.

control, the Alexa-488-labelled SREBP-2 in cells treated with apigenin was found to be limited to the cytosol, and very little of the labelled SREBP-2 was visualised in the nucleus as shown in the DAPI-labelled nuclei (Fig. 3 Merge image). This observation indicated that apigenin prevented the translocation of SREBP-2.

\section{Sterol responsive element-driven luciferase activities and electromobility shift assay}

SREBP-2 transactivation represents the most common regulation for $H M G C R$ expressions. In view of the interference of apigenin on SREBP-2 translocation, the transcriptional control on the downstream gene was evaluated. The SRE-driven luciferase activity was significantly repressed by apigenin at $25 \mu \mathrm{M}$ (Fig. 4(a)). On the other hand, an electromobility shift assay was carried out for investigating the interaction between the N-SREBP-2 and SRE motifs (Fig. 4(b)). The position of the interacting band was revealed by co-incubating with $7 \times \mathrm{SRE}$ unlabelled oligonucleotide fragment or anti-N-SREBP-2. The band was competed out under either treatments. Our data showed that the interaction decreased when apigenin was administered to the WRL- 68 cells.

\section{Status of protein kinases in cells treated with apigenin}

AMP-activated protein kinase. As previous studies have shown that protein kinases might participate in the processing

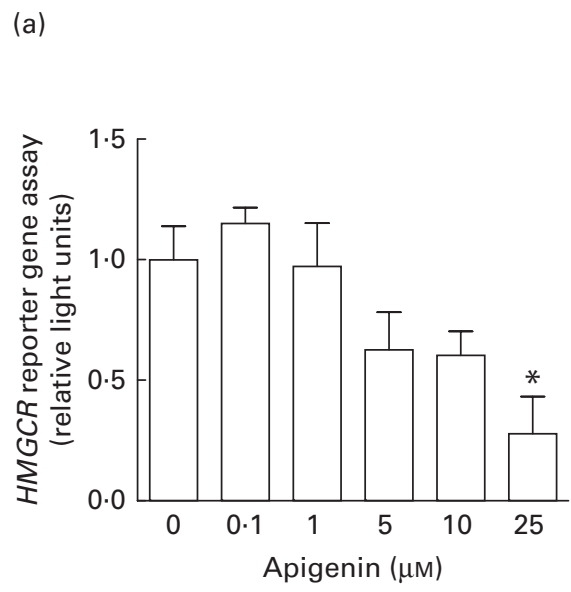

(b)

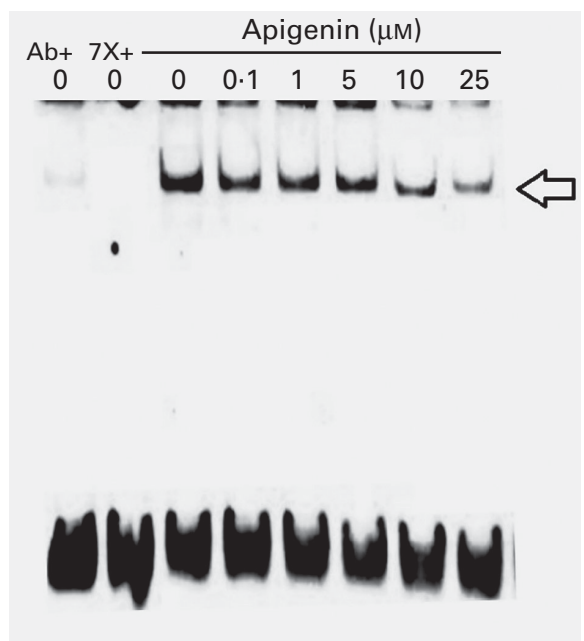

Fig. 4. Effect of apigenin on sterol responsive element (SRE)-driven luciferase activity and SRE-DNA interaction. The hepatic cells WRL-68 were seeded in sixwell culture plates and treated with apigenin at $0,0 \cdot 1,1,5,10,25 \mu \mathrm{M}$. In (a), WRL-68 cells were transiently transfected with a firefly luciferase reporter gene driven by SRE-containing 3-hydroxy-3-methylglutaryl-CoA reductase $(H M G C R)$ fragment $(-1194$ to -49$)$ and a Renilla luciferase control plasmid. The cells were treated with $0,0 \cdot 1,1,5,10,25 \mu \mathrm{M}$-apigenin for $24 \mathrm{~h}$. Values are means, with their standard errors represented by vertical bars $(n 3)$. Mean value was significantly different. (b) The SRE-DNA interaction. After 24-h treatment, nuclear extracts were obtained from the cells and electromobility shift assay was performed. $(\Leftrightarrow)$ is the sterol regulatory element-binding protein-2-SRE interacting band. The image represents one of two independent experiments $(P<0.05)$. 
and activation of SREBP-2, we looked into the status of some protein kinases under apigenin treatment. AMPK is important in regulating the processing of SREBP-2, and it was activated by apigenin as shown in Fig. 5(a). The amount of phosphoAMPK $\alpha$ was increased under 10 or $25 \mu \mathrm{M}$-apigenin treatment, and the total amount of AMPK $\alpha$ was shown right underneath for each treatment. A follow-up study was conducted to show the apigenin-activated AMPK on SREBP-2 processing. The apigenin-reduced cleavage of SREBP-2 was reverted by the AMPK-specific inhibitor compound $\mathrm{C}$ as shown in Fig. 5(b). This result illustrated that AMPK activated by apigenin was involved in the decreased processing of the precursor protein of SREBP-2.

Mitogen-activated protein kinase. The activation status of other protein kinases including some common mitogenactivated protein kinase, were also determined by western analysis (Fig. 5(c)). The result did not reveal any changes in p-JNK or phospho-P38; however, p-ERK $1 / 2$ appeared to be reduced. Because of the interrelationship of SREBP-2 activation, a reduction in ERK could be a part of the transcriptional factor inactivation. According to the optical density analysis (Fig. 5(d)), p-ERK 1/2 appeared to be decreased in cells treated with $\geq 5$ and $10 \mu \mathrm{m}$-apigenin.

(a)

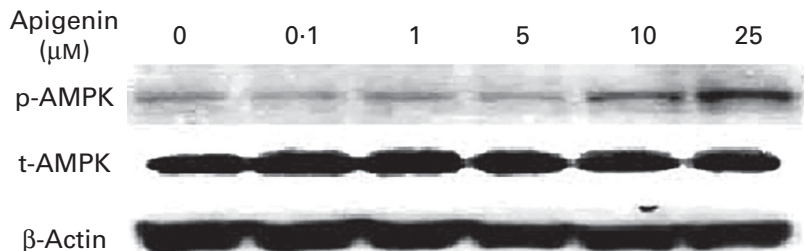

mRNA expression of 3-hydroxy-3-methylg/utaryl-CoA reductase (HMGCR), 3-hydroxy-3-methylglutaryl-CoA synthase (HMGCS) and squalene epoxidase (SQLE)

In view of the decreased transcription of SREBP-2 upon the apigenin treatment, the mRNA expression of $H M G C R$, $H M G C S$ and SQLE were also determined. These are the downstream targets of SREBP-2 and involved in cholesterol synthesis. Real-time RT-PCR showed that apigenin reduced the mRNA levels of the three genes by $>40 \%$ at or above $5 \mu \mathrm{M}$ in WRL-68 cells (Fig. 6(a), (c) and (d)). The suppression on HMGCR was minimal but significant $(P<0.05)$ in cells treated with $1 \mu \mathrm{M}$-apigenin, and the HMGCR protein was also reduced at about $10 \mu \mathrm{m}$ (Fig. 6(b)).

\section{Discussion}

In the present study, we demonstrated that apigenin reduced SREBP-2 nuclear translocation twofold: deterring processing at the post-transcriptional level and suppressing the full-length protein expression. In the vein of post-transcriptional modification, AMPK was activated and the full-length SREBP-2 could not be processed into its active form. Alternatively, the (c)

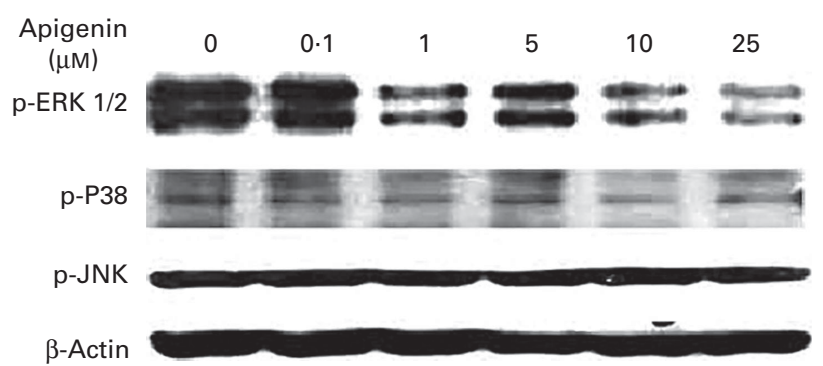

(b)

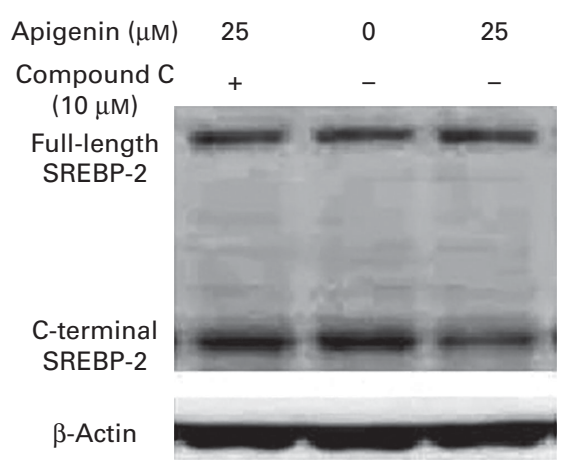

(d)
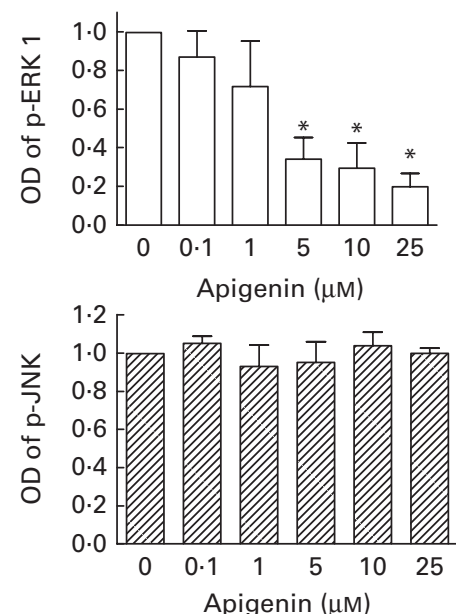
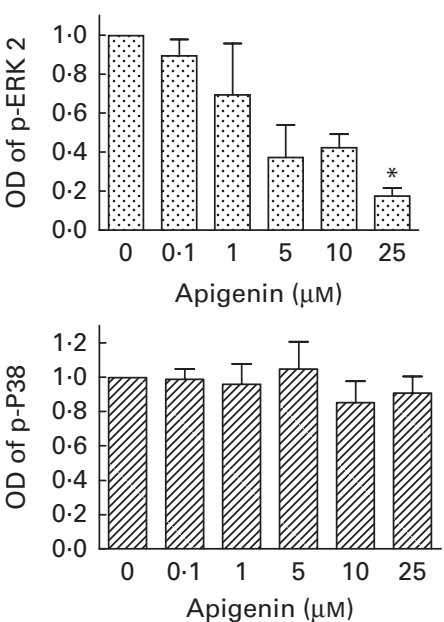

Fig. 5. AMP-activated protein kinase (AMPK) and mitogen-activated protein kinase (MAPK) status in apigenin-treated WRL-68 cells. WRL-68 cells were cultured and were treated with various concentrations of apigenin and co-treated with the AMPK inhibitor Compound C. After $24 \mathrm{~h}$ of treatment, the cell lysates were immuno blotted for AMPK and sterol regulatory element-binding protein-2 (SREBP-2). Images for AMPK and SREBP-2 are displayed in (a) and (b), respectively. In (a), phosphorylated and total AMPK $\alpha$ are labelled as p-AMPK and t-AMPK, respectively. The results represent one of two independent experiments. MAPK were examined in a separate experiment, the cell lysates were immunoblotted for different protein kinases. The image (c) represents one of three independent experiments. The optical density (OD) readings are shown in (d). Values are means, with their standard errors represented by vertical bars $(n 3)$. ${ }^{\star}$ Mean value was significantly different $(P<0.05)$. p-ERK 1/2, phospho-extracelluar signal-regulated protein kinase 1/2; p-JNK, phospho-C-Jun N-terminal kinase. 

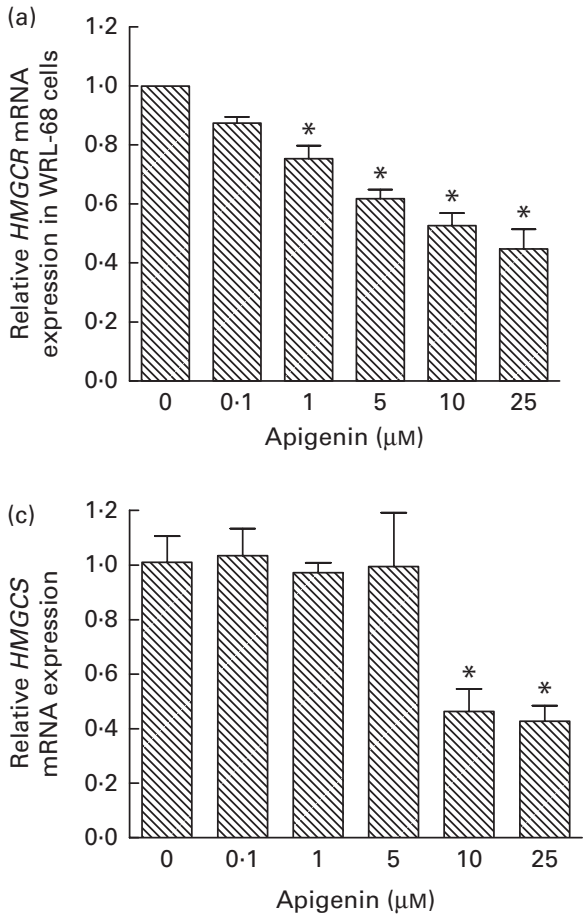
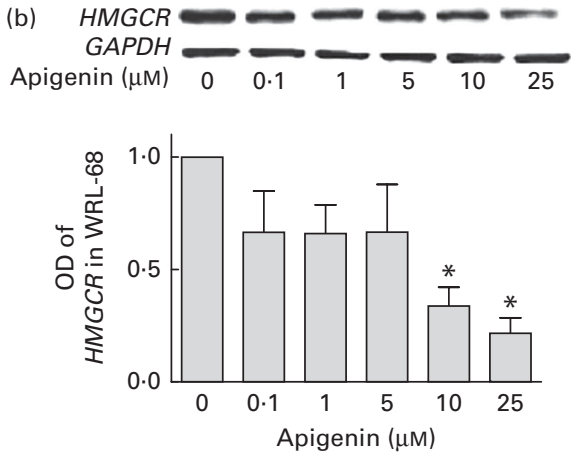

(d)

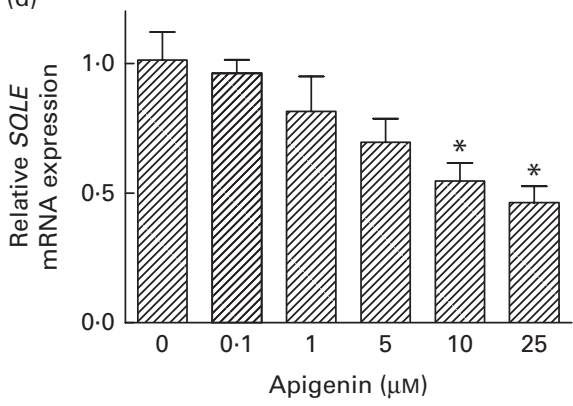

Fig. 6. Expression of 3-hydroxy-3-methylglutaryl-CoA reductase (HMGCR), 3-hydroxy-3-methylglutaryl-CoA synthase (HMGCS), and squalene epoxidase (SQLE) in apigenin-treated WRL-68 cells. WRL-68 cells were treated with various concentrations of apigenin, and cultured for $24 \mathrm{~h}$. mRNA of HMGCR, HMGCS, and SQLE were quantified by real-time RT-PCR and the results are shown in (a), (c) and (d), respectively. Western blot on HMGCR was also performed on the cell cultures under the same treatment. The results are displayed in (b). The image is a representation of three independent experiments, and the optical density (OD) readings are in the lower panel. Values of mRNA expression or OD are means with their standard errors represented by vertical bars $(n 3) .{ }^{*}$ Mean value was significantly different from the control $(0 \mu \mathrm{M})(P<0.05)$.

decreased amount of full-length SREBP-2 protein would limit the mature protein formation. As a result N-SREBP-2 migrating from the cytoplasm to the nucleus would be reduced in both cases. However, the former mechanism appeared to be the major player because the apigenin concentration leading to SREBP-2 protein suppression should exceed the achievable concentration from functional food consumption or dietary supplementation. Ultimately, the transactivation of SRE-bearing genes, such as HMGCR, was compromised, and the mRNA expression was suppressed. SREBP-2 controls the expression of more than sixteen genes which are crucial for cholesterol homeostasis ${ }^{(3)}$. LDLR gene is such a target; nevertheless, we did not observe any significant changes in the transcripts of $L D L R$ under apigenin treatment (data not shown). Since multiple mechanisms have been identified for the regulation of $L D L R$ expression ${ }^{(22)}$, the reduced SREBP-2 could be balanced out by some positive regulators.

AMPK is a key enzyme responsible for cellular energy homeostasis. As reviewed by Steinberg \& Kemp ${ }^{(23)}$, AMPK is a trimeric complex made up of $\alpha$-, $\beta$ - and $\gamma$-subunits. The $\alpha$-subunit is the catalytic domain, whereas $\beta$ - and $\gamma$-subunits are involved in the kinase's regulatory function. This kinase can be activated by phosphorylation at Thr-172 of the $\alpha$-subunit. Upon phosphorylation, a conformational change would expose the catalytic domain, and the enzyme is ready for the binding and conversion of its substrate. Some phytochemicals, the diabetic drug metformin, liver kinase B1 and $\mathrm{Ca} /$ calmodulin-dependent protein kinase kinase $\beta$ are AMPK activators. In the present study AMPK prevented SREBP-2 from processing and this is consistent with a previous investigation demonstrating the interaction between AMPK $\alpha$ and $\operatorname{SREBP}^{(9)}$.

As described earlier, signalling transduction pathways are major regulators of SREBP-2. Contrasting to the SREBP-2 activating capability of ERK $1 / 2$, AMPK negatively regulates the post-transcriptional processing ${ }^{(9)}$. Consistent with a previous study demonstrating that apigenin activates AMPK in cultured hepatocytes ${ }^{(24)}$, our data suggested that the flavone deactivated SREBP-2 through manipulating AMPK and ERK differentially.

Previous studies have described that apigenin may interfere with cholesterol metabolism. As a purified compound or an active ingredient of artichoke, apigenin can block the synthesis of cholesterol in culture cells ${ }^{(25,26)}$. Activation of AMPK could contribute to the blockage ${ }^{(27)}$. Extracts of Salix matsudana leaves, in which apigenin glucoside is a major component, reduce blood cholesterol in mice fed with high fat diet ${ }^{(28)}$. However, none of these studies describes the role of AMPK in the processing of SREBP-2. Our results were consistent with these studies; moreover, the present study offered an insight into the mechanisms of action.

Some non-nutritive chemicals isolated from plant foods have also shown their plasma cholesterol-lowering effect. Plant stanol esters, which deter cholesterol absorption, are functional food ingredients under the brand name Benecol ${ }^{\circledR}$. Other phytochemicals like catechin ${ }^{(27)}$, genistein ${ }^{(29)}$, policosanol $^{(30)}$ 
and hawthorn extracts ${ }^{(31)}$ are inhibitors of the enzyme HMGCR. Some also activate AMPK, which phosphorylates HMGCR and reduces its activity. Previous studies ${ }^{(23)}$ have shown that the phosphorylation status at Ser-872 of HMGCR is critical in determining its enzyme activity. AMPK may introduce phosphorylation at this site and produce a steric hindrance to NADPH binding at the catalytic site. The enzyme activity is then undermined. Recently it was observed that HepG2 cells treated with mulberry anthocyanins display reduced expression of $S R E B P-2$ and $H M G C R$; these effects appear to be associated with increased phosphorylation of $\mathrm{AMPK}^{(32)}$. In the preliminary screening of the present study, apigenin appeared to be the most potent in blocking the SREBP-2 processing by increasing the active form of AMPK. However, the present study did not address the enzymatic activity of HMGCR.

The daily intake of apigenin in another study is about $4 \mathrm{mg}$, the plasma concentration is roughly $0.01 \mu \mathrm{M}$, and the maximum plasma concentration can be as high as $0.4 \mu \mathrm{m}$ at $8 \mathrm{~h}$ after celery leaf consumption ${ }^{(33)}$. Going by the data of the present study, the effective dose of $1 \mu \mathrm{m}$ should fall within the functional food or dietary supplement range of consumption in human beings. According to a pharmacokinetic study in rats ${ }^{(34)}$, oral dosages of $5.4 \mathrm{mg}$ apigenin $/ \mathrm{kg}$ body weight would produce a $C_{\max }$ value of $16.5 \mu \mathrm{m}$ in serum. Besides, apigenin is widely found in traditional herbal medicine. Given its high bioavailability, its action on cholesterol synthesis could be achievable in this administrative method. However, the final drug concentration still relies on the specific herbal composition of prescription.

Statins have been prescribed clinically for controlling blood cholesterol, and they inhibit HMGCR activity. Apigenin, by contrast, might act on the enzyme's upstream transcriptional factor. In conclusion, the present study renewed our understanding of apigenin-SREBP-2 interaction at the post-translational level. These findings also supported the hypothesis that consuming apigenin-rich food may prevent hypercholesterolemia.

\section{Acknowledgements}

This study was supported by a Chinese University of Hong Kong Direct Grant Project (grant no. 4053047).

The authors declare that there are no conflicts of interest.

The author' contributions are as follows: L. K. L. and S.-M. L. were responsible for the conceptualisation of the research and study design; T. Y. W. and S.-M. L. conducted the experiments; all the three authors performed data analysis; L. K. L. and T. Y. W. contributed to the writing of the manuscript.

\section{References}

1. Anum EA \& Adera T (2004) Hypercholesterolemia and coronary heart disease in the elderly: a meta-analysis. Ann Epidemiol 14, 705-721.

2. Horton JD, Goldstein JL \& Brown MS (2002) SREBPs: activators of the complete program of cholesterol and fatty acid synthesis in the liver. J Clin Invest 109, 1125-1131.
3. Rawson RB (2003) The SREBP pathway - insights from Insigs and insects. Nat Rev Mol Cell Biol 4, 631-640.

4. Guan G, Dai P \& Shechter I (1998) Differential transcriptional regulation of the human squalene synthase gene by sterol regulatory element-binding proteins (SREBP) $1 \mathrm{a}$ and 2 and involvement of $5^{\prime}$ DNA sequence elements in the regulation. J Biol Chem 273, 12526-12535.

5. Sakakura Y, Shimano H, Sone H, et al. (2001) Sterol regulatory element-binding proteins induce an entire pathway of cholesterol synthesis. Biochem Biophys Res Commun 286, 176-183.

6. Lewis CA, Griffiths B, Santos CR, et al. (2011) Regulation of the SREBP transcription factors by mTORC1. Biochem Soc Trans 39, 495-499.

7. Edwards PA, Tabor D, Kast HR, et al. (2000) Regulation of gene expression by SREBP and SCAP. Biochim Biophys Acta 1529, 103-113.

8. Kotzka J, Lehr S, Roth G, et al. (2004) Insulin-activated Erk-mitogen-activated protein kinases phosphorylate sterol regulatory element-binding protein-2 at serine residues 432 and 455 in vivo. J Biol Chem 279, 22404-22411.

9. Li Y, Xu S, Mihaylova MM, et al. (2011) AMPK phosphorylates and inhibits SREBP activity to attenuate hepatic steatosis and atherosclerosis in diet-induced insulin-resistant mice. Cell Metab 13, 376-388.

10. Hirano Y, Yoshida M, Shimizu M, et al. (2001) Direct demonstration of rapid degradation of nuclear sterol regulatory element-binding proteins by the ubiquitin-proteasome pathway. J Biol Chem 276, 36431-36437.

11. Paladini AC, Marder M, Viola H, et al. (1999) Flavonoids and the central nervous system: from forgotten factors to potent anxiolytic compounds. J Pharm Pharmacol 51, 519-526.

12. Arai Y, Watanabe S, Kimira M, et al. (2000) Dietary intakes of flavonols, flavones and isoflavones by Japanese women and the inverse correlation between quercetin intake and plasma LDL cholesterol concentration. J Nutr 130, 2243-2250.

13. Anderson JW, Johnstone BM \& Cook-Newell ME (1995) Meta-analysis of the effects of soy protein intake on serum lipids. N Engl J Med 333, 276-282.

14. Hertog MG, Kromhout D, Aravanis C, et al. (1995) Flavonoid intake and long-term risk of coronary heart disease and cancer in the seven countries study. Arch Intern Med 155, $381-386$

15. Janssen K, Mensink RP, Cox FJ, et al. (1998) Effects of the flavonoids quercetin and apigenin on hemostasis in healthy volunteers: results from an in vitro and a dietary supplement study. Am J Clin Nutr 67, 255-262.

16. Surh YJ (2003) Cancer chemoprevention with dietary phytochemicals. Nat Rev Cancer 3, 768-780.

17. Nielsen SE, Young JF, Daneshvar B, et al. (1999) Effect of parsley (Petroselinum crispum) intake on urinary apigenin excretion, blood antioxidant enzymes and biomarkers for oxidative stress in human subjects. Br J Nutr 81, 447-455.

18. Yang CS, Landau JM, Huang MT, et al. (2001) Inhibition of carcinogenesis by dietary polyphenolic compounds. Annu Rev Nutr 21, 381-406.

19. Thiery-Vuillemin A, Nguyen T, Pivot X, et al. (2005) Molecularly targeted agents: their promise as cancer chemopreventive interventions. Eur J Cancer 41, 2003-2015.

20. Al-Jubouri HHF, Al-Jalil BH, Farid I, et al. (1990) The effect of chamomile on hyperlipidemias in rats. J Fac Med Baghdad 32, 5-11.

21. Livak KJ \& Schmittgen TD (2001) Analysis of relative gene expression data using real-time quantitative PCR and the $2^{-\Delta \Delta C_{\mathrm{T}}}$ method. Methods 25, 402-408. 
22. Cho SY, Jun HJ, Lee JH, et al. (2011) Linalool reduces the expression of 3-hydroxy-3-methylglutaryl CoA reductase via sterol regulatory element binding protein-2- and ubiquitindependent mechanisms. FEBS Lett 585, 3289-3296.

23. Steinberg GR \& Kemp BE (2009) AMPK in health and disease. Physiol Rev 89, 1025-1078.

24. Zang M, Xu S, Maitland-Toolan KA, et al. (2006) Polyphenols stimulate AMP-activated protein kinase, lower lipids, and inhibit accelerated atherosclerosis in diabetic LDL receptordeficient mice. Diabetes 55, 2180-2191.

25. Kim KT, Yeo EJ, Cho SG, et al. (2008) Inhibitory effects of naringenin, kaempherol, and apigenin on cholesterol biosyntehsis in HepG2 and MCF-7 cells. Food Sci Biotechnol 17, 1361-1364.

26. Gebhardt R (2002) Inhibition of cholesterol biosynthesis in HepG2 cells by artichoke extracts is reinforced by glucosidase pretreatment. Phytother Res 16, 368-372.

27. Singh DK, Banerjee S \& Porter TD (2009) Green and black tea extracts inhibit HMG-CoA reductase and activate AMP kinase to decrease cholesterol synthesis in hepatoma cells. J Nutr Biochem 20, 816-822.

28. Han LK, Sumiyoshi M, Zheng YN, et al. (2003) Anti-obesity action of Salix matsudana leaves (Part 2). Isolation of anti-obesity effectors from polyphenol fractions of Salix matsudana. Phytother Res 17, 1195-1198.
29. Sung JH, Lee SJ, Park KH, et al. (2004) Isoflavones inhibit 3-hydroxy-3-methylglutaryl coenzyme A reductase in vitro. Biosci Biotechnol Biochem 68, 428-432.

30. Singh DK, Li L \& Porter TD (2006) Policosanol inhibits cholesterol synthesis in hepatoma cells by activation of AMP-kinase. J Pharmacol Exp Ther 318, 1020-1026.

31. Shih CC, Lin CH, Lin YJ, et al. (2013) Validation of the antidiabetic and hypolipidemic effects of hawthorn by assessment of gluconeogenesis and lipogenesis related genes and AMP-activated protein kinase phosphorylation. Evid Based Complement Alternat Med 2013, 597067.

32. Chang JJ, Hsu MJ, Huang HP, et al. (2013) Mulberry anthocyanins inhibit oleic acid induced lipid accumulation by reduction of lipogenesis and promotion of hepatic lipid clearance. J Agric Food Chem 61, 6069-6076.

33. Cao J, Zhang Y, Chen W, et al. (2010) The relationship between fasting plasma concentrations of selected flavonoids and their ordinary dietary intake. Br J Nutr $\mathbf{1 0 3}$, 249-255.

34. Chen Z, Tu M, Sun S, et al. (2012) The exposure of luteolin is much lower than that of apigenin in oral administration of Flos Chrysanthemi extract to rats. Drug Metab Pharmacokinet 27, 162-168. 